\title{
Down-Regulation of Long Non-Coding RNA CASC9 Suppresses the Migration and Invasion of Esophageal Cancer Cells
}

\author{
Maliha Ghaffar ${ }^{1, *, \dagger}$, Sara Khodahemmati ${ }^{1, \dagger}, \operatorname{Jintao} \mathbf{L i}^{1,}{ }^{*}$, Muhammad Shahzad ${ }^{3}$, Minglian Wang ${ }^{1}$, \\ Yangjunqi Wang', Yi Zeng, ${ }^{1,2}$, \\ ${ }^{1}$ Beijing Key Laboratory of Environmental \& Viral Oncology, College of Life Science and Bio-Engineering, Beijing University of \\ Technology, Beijing, China \\ ${ }^{2}$ State Key Laboratory for Infectious Disease Prevention and Control, National Institute for Viral Disease Control and Prevention, Chinese \\ Center for Disease Control and Prevention, Beijing, China \\ ${ }^{3}$ School of Economics and Management, North China Electric Power University, Beijing, China
}

Email address:

malihaghaffar@yahoo.com(M. Ghaffar), ljt2002@qq.com(JintaoLi), zengyicdc@sina.com(Yi Zeng)

${ }^{*}$ Corresponding author

$\dagger$ Maliha Ghaffar and Sara Khodahemmati are co-first authors.

\section{To cite this article:}

Maliha Ghaffar, Sara Khodahemmati, Jintao Li, Muhammad Shahzad, Minglian Wang, Yangjunqi Wang, Yi Zeng. Down-Regulation of Long Non-Coding RNA CASC9 Suppresses the Migration and Invasion of Esophageal Cancer Cells. International Journal of Biomedical Engineering and Clinical Science. Vol. 4, No. 1, 2018, pp. 25-29. doi: 10.11648/j.ijbecs.20180401.15

Received: March 7, 2018; Accepted: March 24, 2018; Published: April 12, 2018

\begin{abstract}
The objective of the study was to investigate the role of CASC9 in esophageal cancer cells migration and invasion. Expression of long noncoding RNA (lncRNA) CASC9 was found high in esophageal cancer cells by qRT-PCR. The CASC9 knockdown effects on cell migration and invasion were analyzed in vitro. Expression of lncRNA CASC9 was found markedly high in KYSE-450 cell line as compared to other esophageal cancer cell lines. After the knockdown of CASC9, migration and invasion were significantly decreased as compared to negative control. Increased expression of CASC9 before knockdown and decreased expression after knockdown in esophageal cancer cells, showed that CASC9 may act as a biomarker for esophageal cancer and may help to understand the mechanisms of this lethal disease.
\end{abstract}

Keywords: CASC9, Esophageal Cancer, Cell Migration, Invasion, Long Noncoding RNA

\section{Introduction}

Esophageal cancer (EC) is one of the deadliest malignancies in the world and ranked as the $6^{\text {th }}$ main cause of cancer deaths $[1,2]$. The two main subtypes of EC are esophageal squamous cell carcinoma (ESCC) and esophageal adreno-carcinoma (EAC). Both types are characterized by distinct pathology and etiology but the most prominent histologic type in China is ESSC as its occurrence is more than $90 \%[1,3]$. EC is diagnosed at late-stage despite the advancement in diagnostic techniques and therapeutics. Overall 5 years survival rate is less than $40 \%$ due to the aggressiveness of this lethal malignancy $[4,5]$. The mortality rate can be reduced by early diagnosis along with effective therapeutics and novel biological markers with high specificity and sensitivity can help us to achieve this goal. For this purpose, it is the need of time to understand all molecular changes which take place in biological pathways as a result of the disease and will give us a better understanding of the disease.

As a result of transcription, a large numbers of proteincoding, as well as non-protein coding RNAs are produced. In 2002, non-coding RNAs were discovered for the first time [6]. Non-coding RNAs are proved to be key regulators of different biological pathways which can cause transformation of normal cells to malignant cells [7,8]. Long non-coding RNAs (lncRNAs) are non-coding RNA molecules consists of more than 200 nucleotides and protein-coding potential is either absent or limited. Research evidence has proved their 
role in cell growth, proliferation, differentiation and other biological processes [9-11]. Aberrant lncRNAs expression is documented by different researchers in different diseases. They cause chromatin modification and regulate transcription as well as translation processes. Along with this, they also regulate expression of different genes. [12-14]. Aberrant expression of lncRNAs is associated with many human diseases especially cancer. Their deregulated expression is tissue-specific as well as cancer specific. They play role in oncogenic pathways as well as tumor suppressing pathways $[14,16]$. This new research direction can help in identification of the cause of different diseases including esophageal cancer.

The first lncRNA identified is H19 which is studied in different cancers and its role is recognized as tumor suppressor gene [17, 18]. POU3F3, HOTAIR, and SPRY4IT1 are some of the important lncRNAs previously investigated as potential prognostic biomarkers [19-21].

In the present study, the expression of CASC9 was analyzed in human Esophageal cancer cell lines KYSE450, EC9706, EC109, and KYSE150 to compare the expression difference between them. Different cell lines were used to investigate the expression of CASC9 while functions of CASC9 in cell invasion and migration were assessed in esophageal cancer cell line KYSE-450.

\section{Materials and Methods}

\subsection{Cell Culture Conditions}

KYSE450, EC9706, EC109, and KYSE150 are the human esophageal cancer cell lines which were used for this study. These cell lines were maintained in DMEM (Hyclone, Thermo Scientific) with $10 \%$ FBS (Gibco, Life Technologies) and $1 \% \mathrm{ps}$. All the cell lines were maintained in an incubator set at $37^{\circ} \mathrm{C}$ with $5 \% \mathrm{CO}_{2}$.

\subsection{RNA Extraction and $q R T-P C R$}

Total RNA was extracted from Cell lines by using Trizol (Invitrogen, Life Technologies) according to manufacturer's protocol and DNA was removed by DNase kit (Promega). Reverse Transcription kit (Promega) was used for cDNA synthesis. Applied BiosystemsViiA ${ }^{\text {TM }} 7$ (Life Technologies) was used for quantitative real-time PCR (qRT-PCR) with SYBR Select Master Mix (Life Technologies). 384 well block plate was used for qRTPCR and each well contains $2 \mathrm{ul} \mathrm{cDNA}$ in a total volume of 8ul. Primers for qRT-PCR were designed by Beacon Designer 7 Software. GAPDH was used as an endogenous control for the relative expression levels of CASC9. Each sample was used in triplicates. Denaturation was done at $95^{\circ} \mathrm{C}$ for $10 \mathrm{~min}$, then $40 \mathrm{PCR}$ cycles at $95^{\circ} \mathrm{C}$ for $15 \mathrm{sec}$ and $60^{\circ} \mathrm{C}$ for one min.

\subsection{Transfection}

Two small interfering RNAs (siRNAs) and one negative control (si-NC) were synthesized (RiboBio, China) for silencing CASC9. $20 \times 10^{4}$ cells per well were seeded on sixwell plates after trypsinization. After 24 hours, cells were transfected with two siRNAs and si-NC with Transfection Reagent FuGENE HD (Promega). Interfering efficiency of siRNA was checked by qRT-PCR after 48 hours of transfection. The siRNAs with higher silencing efficacy was used for migration and invasion experiments.

\subsection{Transwell Cell Migration and Invasion Assay}

Costar chambers (Corning Incorporated, USA) with transwell inserts, having $8 \mu \mathrm{m}$ pore size were used for cell migration and invasion assay. $1 \times 10^{6}$ cells per $\mathrm{mL}$, transfected with si-CASC9 or si-NC were suspended in the serum-free medium after trypsinization and $100 \mu \mathrm{L}$ were seeded in the upper chamber. The upper chambers for invasion assay were already coated with Matrigel.750uL DMEM with 15\% FBS was added to the lower chamber. Then cell invasion and migration chambers were placed in the incubator for one day. After incubation invaded and migrated cells were fixed with ethanol and stained with crystal violet $(0.1 \%)$. Cells in the upper chamber were carefully removed by cotton swab. Then invaded and migrated cells were photographed and counted at 3-4 different fields under an inverted microscope.

All biological experiments were performed in triplicate and data is presented as mean.

\section{Results}

\subsection{Expression of CASC9 in Different Cell Lines}

Expression of CASC9 was measured by qRT-PCR. KYSE 450 cell line showed the highest level of CASC9 expression as compared to other esophageal cancer cell lines used here (Figure 1).

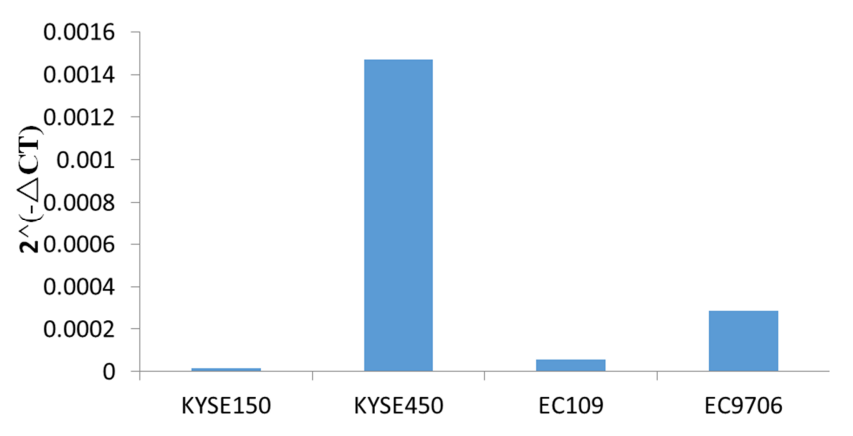

Figure 1. Expression of lncRNA CASC9 in different esophageal cancer cells.

\subsection{Expression of CASC9 after Knockdown}

We down-regulated the expression of CASC9 with the help of its knockdown expression in KYSE-450 cell line, using two small interfering RNA (siRNA) and one negative control siRNA (si-NC). The efficiency of the siRNA was determined by qRT-PCR, after 48 hours of transfection. siRNA with higher knockdown efficiency was used for further experiments (Figure 2). 


\subsection{Transwell Cell Migration and Invasion Assay}

Transwell invasion and migration assays were performed to investigate the role of CASC9 in the regulation of cell migration and invasion in human esophageal cancer cells KYSE-450. Transwell cell migration and invasion assay showed that the invasion and migration ability of esophageal cancer cells transfected with si-CASC9 was markdly suppressed as compared to the si-NC group (Figure 3 \& 4).

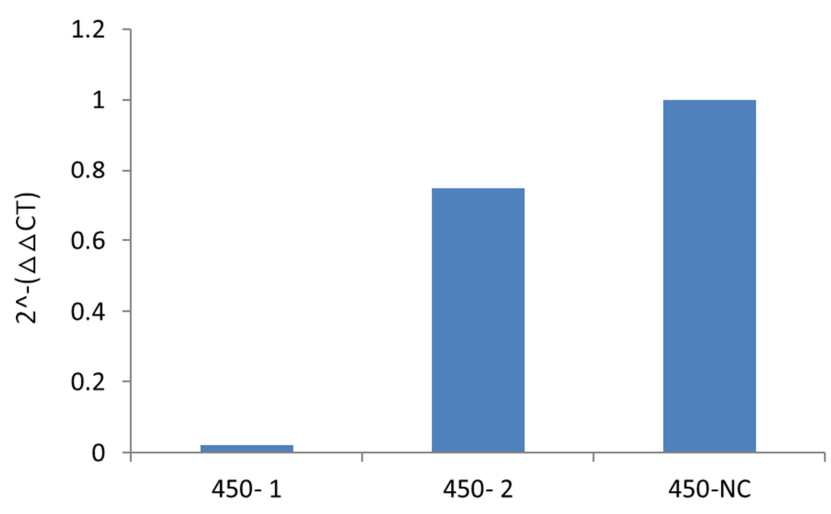

Figure 2. Expression of CASC9 after knockdown in KYSE-450.

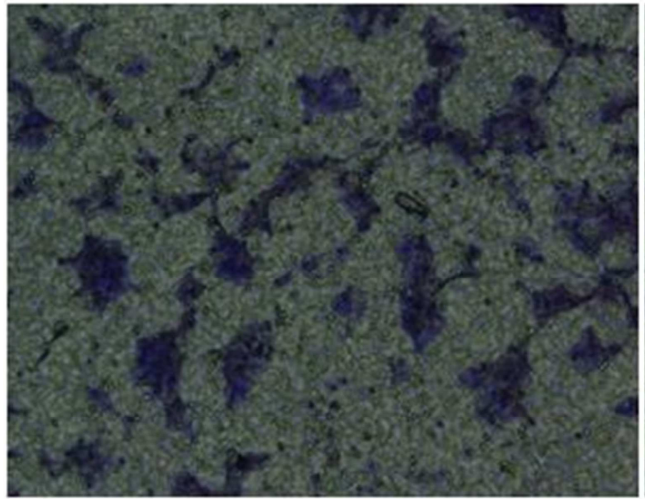

si- NC

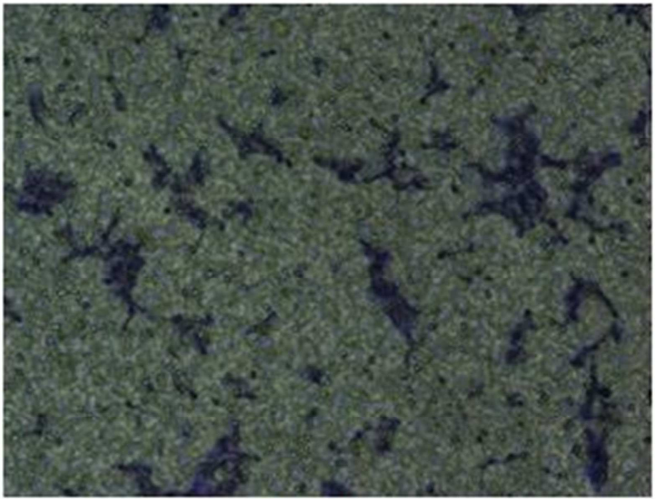

si- CASC9

(a)

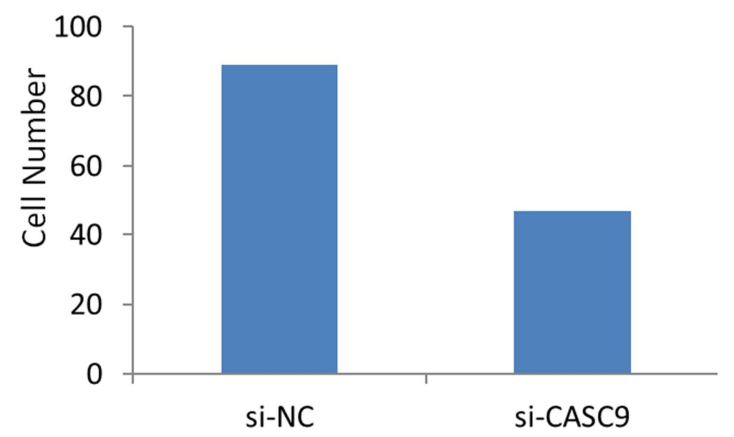

(b)

Figure 3. Cell migration capacity decreases after knockdown of CASC9 as compared to NC in KYSE-450 cells.

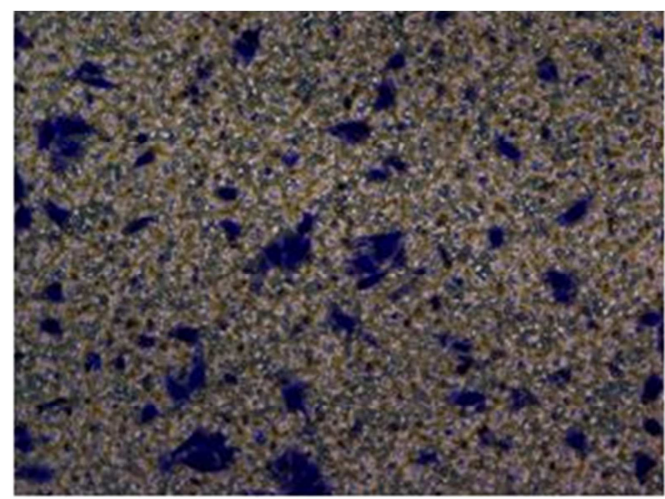

si-NC

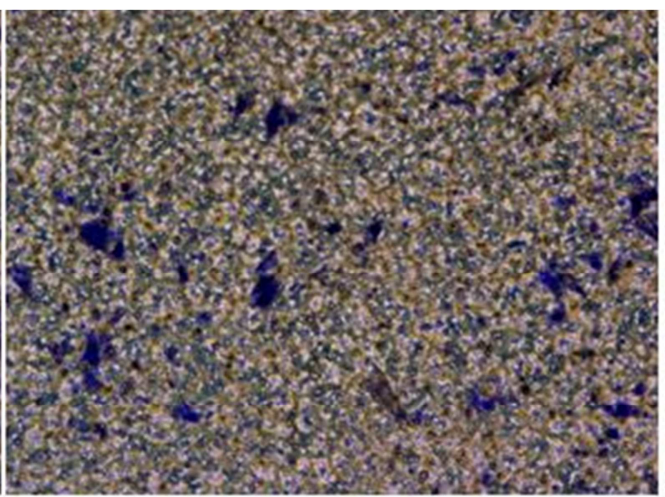

si-CASC9

(a) 


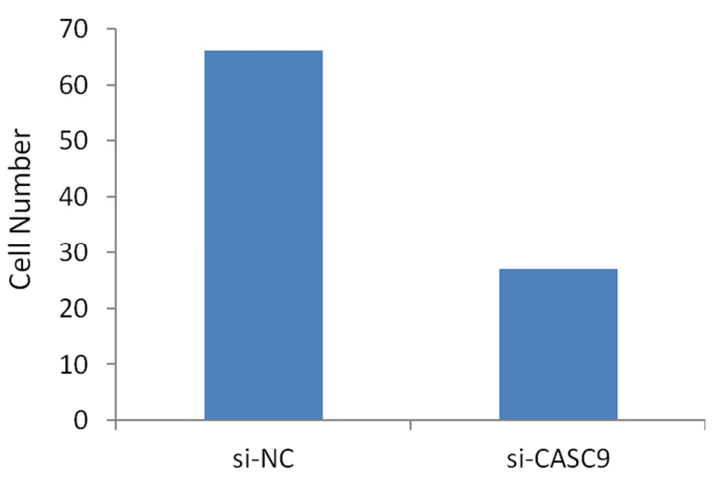

(b)

Figure 4. Transwell invasion assay shows suppression after knockdown of CASC9 as compared to NC in KYSE-450 cells.

\section{Discussion}

Esophageal cancer is one of the main cause of cancer deaths $[1,2]$. It is the need of time to find some new molecular regulators for early diagnosis and treatment. Accumulating data in the recent years is providing evidence and showing the importance of regulatory RNAs which include protein-coding as well as non-protein coding RNAs.

In the recent decade, many lncRNAs are associated with different diseases especially cancers. We know about them as biological regulators but their detailed functions are still not known. The knowledge about lncRNA is very little especially their role in the progression of esophageal cancer. Aberrant expression of lncRNAs is documented in different cancers $[12,14]$.

The up-regulated expression of CASC9 in esophageal cancer cell lines was found and its highest expression was found in KYSE-450. The previous study about CASC9 has found its high expression in about $65 \%$ of the tissue samples [22]. To the best of our knowledge, it is the first study exploring the functions of CASC9 in KYSE-450. High expression CASC9 was found in Kyse150 and TE1 cell lines in one of the previous study [23]. In this study, the main focus was to find out the role of CASC9 in cell migration and invasion. The highest expression was found in KYSE450 through qRT-PCR. After this, siRNA mediated knockdown of lncRNA CASC9 showed a marked decrease in cell migration and invasion ability. The result shows that knockdown of lncRNA CASC9 may decrease esophageal cancer migration and invasion ability in vitro. These results demonstrate that CASC9 may have an important contribution to progression and development of esophageal cancer.

The present research shows that lncRNA CASC9 may act as a novel biomarker in esophageal cancer and its further functions can be studied by using KYSE-450 cell line. However, there is still need to further explore the mechanisms involved in CASC9-mediated cell migration and invasion of esophageal cancer cells. There is need of much more research for understanding of its functions and mechanisms involved in EC progression which can help to use CASC9 as a therapeutic target.

The limitation of the present study is that the evaluation of role of CASC9 in EC progression was done only by using esophageal cancer cell lines therefore further extensive research is required to evaluate its other functions by using EC patients' tissue specimens and normal adjacent tissues along with EC cell lines. So the present study provides some basic information about the function of IncRNA CASC9 in EC cell line, KYSE-450.

\section{Conclusions}

In conclusion, the findings of this research indicate that CASC9 may be related to esophageal cancer pathogenesis and progression. The results suggest that IncRNA CASC9 may be proved as a novel biomarker and a therapeutic target for EC. For this purpose, there is need to understand the detailed functions of CASC9 and mechanisms involved in EC progression.

\section{Acknowledgements}

This research was financially supported by the China Scholarship Council (CSC) and Beijing Natural Science Foundation (5162003).

\section{References}

[1] DomperArnal MJ, Ferrández Arenas Á, LanasArbeloa Á. (2015). Esophageal cancer: Risk factors, screening and endoscopic treatment in Western and Eastern countries. World J Gastroenterol. 21 (26):7933-43.

[2] Pennathur A, Gibson MK, Jobe BA, Luketich JD. (2013). Oesophageal carcinoma. Lancet. 381:400-412.

[3] Yang L, Parkin DM, Ferlay J, Li L, Chen Y. (2005). Estimates of cancer incidence in China for 2000 and projections for 2005. Cancer Epidemiol Biomarkers Prev. 14 (1):243-50.

[4] Pohl H and Welch HG. (2005). The role of overdiagnosis and reclassification in the marked increase of esophageal adenocarcinoma incidence. J Natl Cancer Inst. 97; 2: 142-146.

[5] Toh Y, Egashira A, Yamamoto M. (2013). Epigenetic alterations and their clinical implications in esophageal squamous cell carcinoma. Gen ThoracCardiovasc Surg. 61:262-269. 
[6] Okazaki Y, Furuno M, Kasukawa T et al. (2002). Analysis of the mouse transcriptome based on functional annotation of 60,770 full-length cDNAs. Nature. 420:563-573.

[7] Mattick JS. (2004). RNA regulation: a new genetics. Nat Rev Genet 4:316-323.

[8] Guttman M, Donaghey J, Carey BW et al. (2011). lincRNAs act in the circuitry controlling pluripotency and differentiation. Nature. 477 (7364):295-300.

[9] Pauli A, Rinn JL, and Schier AF. (2011). Non-coding RNAs as regulators of embryogenesis. Nat Rev Genet. 12:136-149.

[10] Prensner JR and Chinnaiyan AM. (2011). The emergence of IncRNAs in cancer biology. Cancer Discov 1:391-407.

[11] Spizzo R, Almeida MI, Colombatti A, and Calin GA. (2012). Long non-coding RNAs and cancer: a new frontier of translational research. Oncogene. 31:4577-4587.

[12] Mercer TR, Dinger ME, Sunkin SM, Mehler MF, Mattick JS. (2008). Specific expression of long noncoding RNAs in the mouse brain. ProcNatlAcadSci, USA. 105 (2):716-21.

[13] Mercer TR, Dinger ME, Mattick JS. (2009). Long non-coding RNAs: insights into functions. Nat Rev 10 (3):155-9.

[14] Li X, Wu Z, Fu X, Han W. (2013). Long noncoding RNAs: insights from biological features and functions to diseases. Med Res Rev 33 (3):517-53.

[15] Kotake Y, Nakagawa T, Kitagawa K et al. (2011). Long noncoding RNA ANRIL is required for the PRC2 recruitment to and silencing of p15 (INK4B) tumor suppressor gene. Oncogene. 30:1956-1962.
[16] Werven FJ van, Neuert G, Hendrick N et al. (2012). Transcription of two long noncoding RNAs mediates matingtype control of gametogenesis in budding yeast. Cell. 150, $1170-1181$.

[17] Tanos V, Ariel I, Prus D et al. (2004). H19 and IGF2 gene expression in human normal, hyperplastic, and malignant endometrium. Int J Gynecol Cancer. 14:521-525.

[18] Barsyte-Lovejoy D, Lau SK, Boutros PC et al. (2006). The cMyc oncogene directly induces the H19 noncoding RNA by allele-specifi binding to potentiate tumorigenesis. Cancer Res. 66:5330-5337.

[19] Tong YS, Wang XW, Zhou XL et al. (2015). Identification of the long non-coding RNA POU3F3 in plasma as a novel biomarker for diagnosis of esophageal squamous cell carcinoma. Mol Cancer. 14:3.

[20] Li X, Wu Z, Mei Q, Guo M, Fu X, Han W. (2013). Long noncoding RNA HOTAIR, a driver of malignancy, predicts negative prognosis and exhibits oncogenic activity in oesophageal squamous cell carcinoma. $\mathrm{Br} \mathrm{J}$ Cancer. 109:2266-78.

[21] Xie HW, Wu QQ, Zhu B et al. (2014). Long noncoding RNA SPRY4-IT1 is upregulated in esophageal squamous cell carcinoma and associated with poor prognosis. Tumour Biol. $35: 7743-54$.

[22] Hao Y, Wu W, Shi F, Dalmolin RJ, et al. (2015). Prediction of long noncoding RNA functions with co-expression network in esophageal squamous cell carcinoma. BMC Cancer. 15:168.

[23] Pan Z, Mao W, Bao Y, et al. (2016). The long noncoding RNA CASC9 regulates migration and invasion in esophageal cancer. Cancer Med. 5 (9):2442-7. 